\title{
A coplanar probe design for humidity spot measurements of human brain tissue
}

\author{
Tobias Reinecke ${ }^{1}$, Lars Hagemeier2, Sebastian Ahrens ${ }^{1}$, Sven Gitte ${ }^{1}$, Michael Klintschar2, \\ Stefan Zimmermann ${ }^{1}$ \\ ${ }^{1}$ Leibniz University Hannover, Institute of Electrical Engineering and Measurement Technology, \\ Department of Sensors and Measurement Technology, Appelstr. 9A, 30167 Hannover, Germany, \\ reinecke@geml.uni-hannover.de \\ 2 Hannover Medical School, Institute of Forensic Medicine, Carl-Neuberg-Str. 1, 30625 Hannover, \\ Germany
}

\begin{abstract}
:
In an autopsy, the quantification of edema in human brain tissue is helpful to verify the cause of death, especially in case of no visible evidence, or multiple affections. However, there is no standardized procedure defined in forensic medicine for the determination of the water content in parenchymatous organs, such as the brain. Thus, the aim of our work is the development of a fast and exact, but lowcost, measuring system, which fits into the procedure of a routine autopsy. Therefore, the dependency of the permittivity from the water content is utilized. To avoid any brain tissue sampling process a coplanar probe is designed. Thus, it is possible to measure rapidly the tissue permittivity and subsequently the water content by simply pressing it on the tissue. This paper focuses on a new coplanar probe with a reduced sensing area size to enable real spot measurements of the highly inhomogeneous human brain tissue. We compare the results to measurements with a former used coplanar probe and to reference measurements with a commercially available moisture analyzer.
\end{abstract}

Key words: dielectric spectroscopy, permittivity of human brain tissue, coplanar probe, quantification of edema.

\section{Introducion}

Investigating the correlation between brain hydration and agony, Bogomolov et al. found that a slow death is usually accompanied with hyperhydration in the brain [1]. Hyperhydration is observed because during agony, the brain is stressed and as reaction to the stress, the brain expands and creates edema in the tissue. Oppositely a fast death causes all bodily functions to stop immediately and therefore, no or only little edema is found in the brain tissue. Therefore, the hydration state of the brain can be used in a forensic examination as a marker leading to a sound diagnosis of the cause of death. However, until today there is no measuring technique available for the quantitative analysis of edema in the brain tissue. The current practice for estimating the degree of brain hydration is the examination of brain weight, turgor due to flattening of brain convolution, reduction of furrows of the brain and ventricle compression. The findings strongly depend on the investigators experience and are naturally prone to errors.

There are approaches to be found in literature to estimate the degree of edema by radiological examinations. Hereby, Computer Tomography (CT) is of low accuracy due to imprecise theoretical hypotheses [2,3]. Magnetic resonance imaging (MRI) allows a more detailed examination of water-rich tissues, especially in the brain $[4,5]$. However, MRI has three major disadvantages: MRI systems are very expensive and usually not available in forensic institutes. Quantifying the water content takes about 30 minutes and thus, it is very time consuming. Additionally, this method is temperature sensitive [6] and the available sequences are designed for in vivo measurements at normal body temperature and not for the generally lower and inconstant temperature of deceased. Even though recent research activities in CT and MRI show, that the evaluation of the water content is generally possible, the procedure is far off daily routine use $[7,8]$.

One method for the humidity measurement of small tissue samples of parenchymal organs is the tissue desiccation or oven dry method. Here, the humidity content is calculated from the weight difference before and after drying the sample. The drying process runs in a desiccator, which takes about 24 to 60 hours $[9,10]$. To accelerate the drying process the sample can be heated by microwaves [11]. This method is very exact but has the major drawback of bulky and expensive equipment as well as measuring 
times of about $10 \mathrm{~min}$ for one sample, which makes sequential measurements of several samples very time consuming.

Therefore, the aim of our work is the development of a measuring technique for the fast and exact quantification of edema in human brain tissue that is applicable in a routine forensic autopsy. Thinking of the development of a handheld device, one approach could be the measurement of the water dependent conductivity of the tissue. But due to the anisotropic behavior of the tissue conductivity [12], conductivity measurements are expected to perform insufficiently. Therefore, we investigated the quantitative dependency between the relative permittivity $\varepsilon_{r}$ of brain tissue and the water content in the tissue via dielectric measurements using a coaxial measuring chamber [13]. Here, we could derive a linear function linking the real part of the relative permittivity to the water content in the brain tissue. Furthermore, the maximum error for determining the water content from a permittivity measurement with this setup was calculated to $2 \%$. However, the necessary sampling process for introducing the tissue sample into the measuring chamber leads to long measuring times, which makes sequential measurements during autopsy too time consuming, and thus, not applicable. Subsequently, we tested a procedure for dielectric measurements without any sampling process - the open-ended coaxial probe [14]. Here, the probe is pressed on the specimen and the permittivity is determined via a reflection measurement. We could confirm the linear dependency between the real part of the relative permittivity and the water content in the tissue. A maximum error of $3 \%$ is determined for the prediction of the water content from a permittivity measurement with the open-ended coaxial probe. However, this method proved to be very sensitive for variations of the contact pressure. Additionally, the electrical conductivity of the tissue surface has an impact on the accuracy. Furthermore, the necessary calibration of the setup is inconvenient. To calibrate the setup, measurements on materials with known permittivity have to be performed on a regular basis. Hereby, it is required that the permittivity of the calibration materials is close to the specimen permittivity. Thus, mixtures of liquids are used in order to obtain the required permittivity values.

In [15] we present a preliminary version of a coplanar probe that combines the advantages of both former used methods. As for the coaxial measuring chamber, the coplanar probe can be easily calibrated via measurements on commercially available calibration standards. In addition, no sampling is necessary to perform the dielectric measurement. It is simply pressed on the specimen like the open-ended coaxial probe. With this preliminary setup, we could proof that an accurate water content determination is possible. Therefore, in a subsequent work we developed the probe depicted in fig. 1.

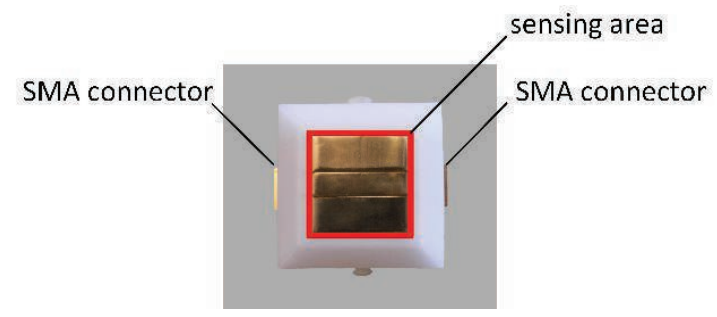

Fig. 1: Coplanar probe with sensing area of $400 \mathrm{~mm}^{2}$.

However, the depicted probe has a sensing area with a size of $400 \mathrm{~mm}^{2}$. Due to the inhomogeneity of the brain tissue there is sometimes a need for smaller spot measurements. That is why we developed a further probe with a reduced sensing area size of $100 \mathrm{~mm}^{2}$. In this paper, we compare the results of both probes via measurements on water ethanol mixtures and human brain tissue.

\section{Theory}

Fig. 2 depicts a cross-section of a coplanar probe. It consists of a dielectric substrate with conductors on the surface. A narrow gap separates the inner conductor from two ground planes on either side. The structure is placed on a specimen. As can be seen from a simulation with CST Microwave Studio 2014, the electromagnetic field is partly inside the substrate and partly inside the specimen.

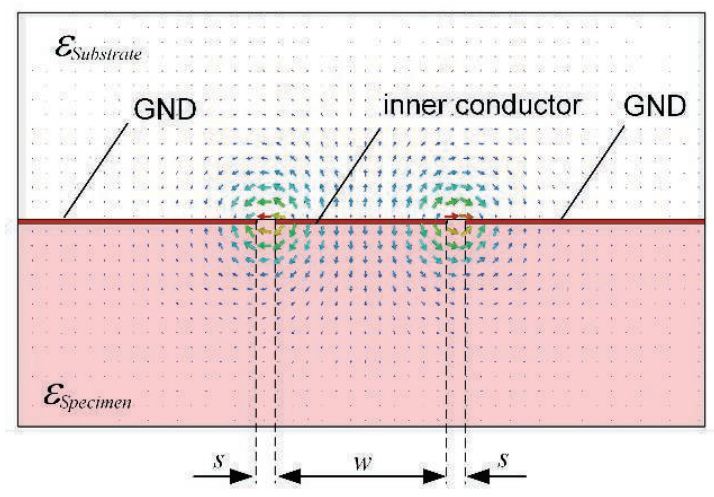

Fig. 2: Schematic and simulated electrical field of a coplanar waveguide. 
As shown in [16], the wave impedance of this structure can be expressed as

$$
\mathrm{Z}=\frac{30 \pi}{\sqrt{\varepsilon_{\mathrm{eff}}}} \frac{\mathrm{K}\left(\mathrm{k}_{\infty}^{\prime}\right)}{\mathrm{K}\left(\mathrm{k}_{\infty}\right)} .
$$

Here, $\mathrm{k}_{\infty}^{\prime}$ and $\mathrm{k}_{\infty}$ are the modulus of the elliptic integral $\mathrm{K}$. Their value is determined by the geometric parameters of the structure, i.e. the width of the inner conductor $w$ and the width of the gap $s$. For the calculation of the structures Impedance, the elliptic integral $\mathrm{K}$ is solved using Matlab. The third parameter determining the Impedance of a coplanar waveguide is the effective permittivity $\varepsilon_{e f f}$, which is a superposition of the substrate permittivity $\varepsilon_{\text {substrate }}$ and the specimen permittivity $\varepsilon_{\text {specimen }}$. Since the specimen permittivity is depending on the water content in the specimen, there is a correlation between the water content and the effective permittivity as well. For the determination of the effective permittivity, a transmission measurement is performed. The reflection of an electromagnetic wave at the interface between connecting cables and coplanar probe can be described with the refection coefficient

$$
\Gamma=\frac{z_{1}-Z_{0}}{Z_{1}+Z_{0}} .
$$

The impedance of the connecting cable is $Z_{0}$ and the Impedance of the coplanar probe is $Z_{1}$. With an Impedance of the unloaded probe in air equal to the impedance of the connecting cables and eq. 1 , the reflection coefficient can be expressed as

$$
\Gamma=\frac{\sqrt{\frac{\varepsilon_{r, \text { eff }}(\text { substrate\&air })}{\varepsilon_{r, \text { eff }}(\text { substrate\&specimen })}}-1}{\sqrt{\frac{\varepsilon_{r, \text { eff }}(\text { substrate\&air })}{\varepsilon_{r, \text { eff }}(\text { substrate\&specimen })}}+1} .
$$

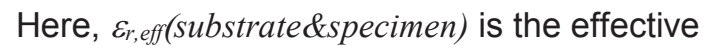
permittivity for the coplanar probe pressed on the brain tissue. $\varepsilon_{r, e f f}$ (substrate\&air) is the effective permittivity for the coplanar probe in air. It needs to be determined via a calibration measurement with the unloaded probe. The transmission of an electromagnetic wave is described by the transmission coefficient

$$
z=e^{-j \cdot \frac{\omega}{c_{0}} \cdot \sqrt{\varepsilon_{r, e f f}} \cdot l} .
$$

In [17] it is shown that with $\Gamma$ and $z$, the complete transmission including multiple reflections is given by

$$
S_{21}=\frac{z \cdot\left(1-\Gamma^{2}\right)}{1-z^{2} \Gamma^{2}}
$$

The so-called Scattering Parameter $S_{21}$ can be measured with a Vector Network Analyzer (VNA). We use a low-cost VNA the VWNA3 by
SDR kits. Since $\Gamma$ and $z$ are both functions of the effective permittivity, eq. 5 connects the measurement value with the effective permittivity. Formulating eq. 5 as root finding problem to

$$
\frac{z \cdot\left(1-\Gamma^{2}\right)}{1-z^{2} \Gamma^{2}}-S_{21}=0
$$

it can be solved numerically [18] with the newton algorithm.

\section{Results}

In a first step, the new probe with a sensing area of $100 \mathrm{~mm}^{2}$ is compared to the probe with a sensing area of $400 \mathrm{~mm}^{2}$ via measurements of water ethanol mixtures. Both sensors are manufactured on a Polyoxymethylene (POM) substrate with a relative permittivity of $\varepsilon_{r}=3.8$. The strip lines are made of gold-coated copper on a thin Polyimide foil. The gap between inner conductor and ground plane is $\mathrm{s}=0.44 \mathrm{~mm}$ and the width of the inner conductor is $w=4.5 \mathrm{~mm}$. With these dimensions, the impedance of unloaded coplanar probe in air $\left(\varepsilon_{\text {r,air }}=1\right)$ is matched to the impedance of the connecting cables with $Z_{0}=50 \Omega$ and no unwanted reflections occur.

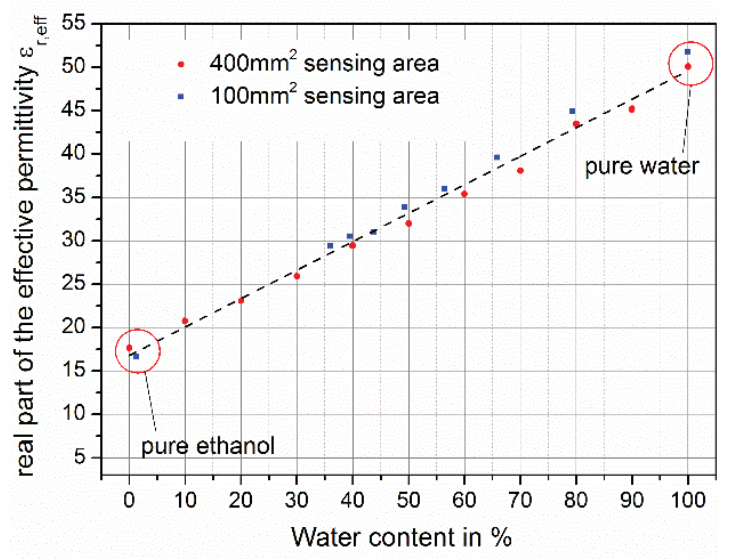

Fig. 3: Verification of the spot measuring coplanar probe via measurements of water ethanol mixtures.

Fig. 3 depicts the results from the verification measurements of water ethanol mixtures. Both sensors produce virtually the same values for the effective permittivity. Deviations can be explained by noise on the measurement values and the temperature dependency of the permittivity of water ethanol mixtures, which is expected to have a higher influence. Therefore, it can be assumed that both sensors will perform equally by measuring brain tissue.

Subsequently, the new probe is tested in a clinical trial. The test is performed under real clinical conditions, i.e. the probe is pressed 
manually on the tissue sample and no tripod and pressure control is used, as it is necessary for the open-ended coaxial probe described in [14]. The water content in the tissue is determined with a commercially available moisture analyzer, the Smart System5 by CEM working with the oven dry method. It has a standard deviation of $\sigma=0.1 \%$. The results are shown in Fig. 4. As shown for the preliminary setup described in [14], there is a linear dependency between the real part of the effective permittivity and the water content. The maximum error for the determination of the water content from a permittivity measurement is determined to $2.5 \%$ for the coplanar probe with a sensing area of $400 \mathrm{~mm}^{2}$.

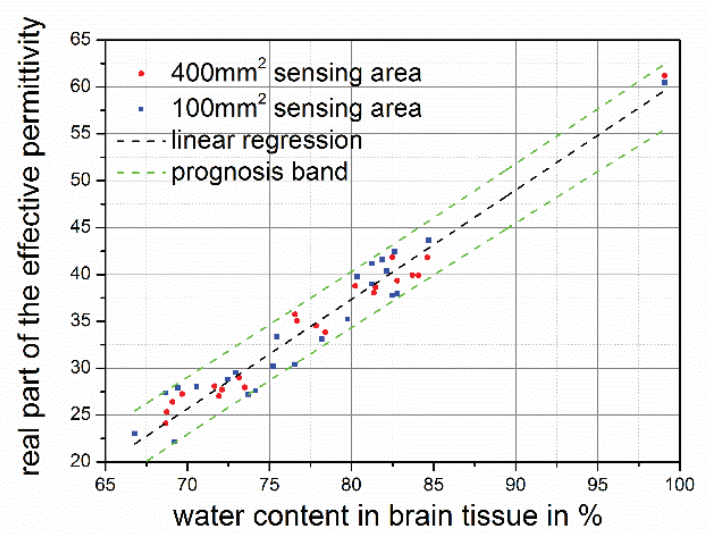

Fig. 4: Linear dependency between the relative humidity in brain tissue and real part of the relative permittivity measured with coplanar probes with different sensing area sizes.

The measurement values determined with the coplanar probe with smaller sensing area are all inside the prognosis band from the former used sensor with the bigger sensing area. However, the scattering of the data is slightly higher which is most certainly caused by a higher error of the commercially available moisture analyzer by CEM. The measured area in the tissue becomes very small using the new probe. Thus, the measured mass of brain tissue is with approximately $0.5 \mathrm{~g}$ far under the minimum mass for accurate measurements of $1.5 \mathrm{~g}$ specified in [19] for the moisture analyzer by CEM.

\section{Conclusion}

In this work, we present measurements with a coplanar probe with a reduced sensing area size compared to a former used probe. This new probe enables measurements for the quantification of edema in human brain tissue in very small areas. This is necessary due to the inhomogeneous nature of the human brain. All measurements where performed under real clinical conditions and compared to the former used sensors and to reference measurements with a commercially available moisture analizer. It could be shown that an accurate determination of the water content in small spots in the human brain tissue is possible with a coplanar probe.

\section{References}

[1] D.V. Bogomolov, O.V. Holzhansky, D.P. Kalashnikov, A.V. Luzin: "Quantitative assessment of brain hydration in determination of death speed and type of tanatogenesis in forensic medical practice". Sud Med Ekspert 2006; 49(1):14-16.

[2] [P.P. Fatouros, Marmarou: "Use of magnetic resonance imaging for in vivo measurements of water content in human brain: method and normal values". J Neurosurg. 1999; 90:109-115.

[3] [R. Bullock, R. Smith, J. Favier, M. du Trevou, G. Blake: "Brain specific gravity and CT scan density measurements after human head injury". J Neurosurg. 1985; 63(1): 64-68.

[4] C. Ayata, A.H. Ropper: "Ischaemic brain oedema”. J Clin Neurosci. 2002; 9(2):113-124.

[5] T. Neumann-Haefelin, M.E. Moseley, G.W. Albers: "New magnetic resonance imaging methods for cerebrovascular disease: emerging clinical applications", Ann Neurol. 2000; 47(5):559-570.

[6] H. Neeb H, K. Zilles, N.J. Shah: "A new method for fast quantitative mapping of absolute water content in vivo". Neuroimage. 2006; 31(3):11561168.

[7] B.M. Ellingson, T.F. Cloughesy, A. Lai, P.L. Nghiemphu, S. Lalezari, T. Zaw, K. Motevalibashinaeini, P.S. Mischel, W.B. Pope: "Quantification of edema reduction using differential quantitative T2 (DQT2) relaxometry mapping in recurrent glioblastoma treated with bevacizumab". J Neurooncol. 2012; 106:111-119.

[8] B. Volbers, D. Staykov, I. Wagner, A. Dörfler, M. Saake, S. Schwab, J. Bardutzky: "Semiautomatic volumetric assessment of perihemorrhagic edema with computed tomography". Eur J Neurol. 2011; 18(11):13231328.

[9] M.T. Harting, C.T. Smith, R.S. Radhakrishnan, K.R. Aroom, P.K. Dash, B. Gill, C.S. Jr. Cox: "Regional differences in cerebral edema after traumatic brain injury identified by impedance analysis". J Surg Res. 2010; 159(1):557-564.

[10] A. Marmarou, K. Tanaka, K. Shulman: "An improved gravimetric measure of cerebral edema". J Neurosurg. 1982; 56:246-253

[11] M.J. Collins: "Analytical method for determining volatiles, solids and solvent extractables". US Patent No. 4753889 A; 1988.

[12] J. Damez, S. Clerjon, S. Abouelkaram, J. Lepetit: "Beef meat electrical impedance spectroscopy and anisotropy sensing for non-invasive early assessment of meat ageing". Journal of Food Engineering 2008; 85:116-122 
[13] T. Reinecke, L. Hagemeier, V. Schulte, M. Klintschar, S. Zimmermann, Quantification of edema in human brain tissue by determination of electromagnetic parameters, Proceedings, IEEE Sensors, pp. 1390-1393, 2013.

[14] T. Reinecke, L. Hagemeier, H. Spehlbrink, S. Guenther, M. Klintschar, S. Zimmermann, Openended coaxial probe for the quantification of edema in human brain tissue, Sensors and Actuators B, vol. 204, pp. 763-769, 2014.

[15] T. Reinecke, L. Hagemeier, S. Ahrens, M. Klintschar and S. Zimmermann (2014): Permittivity measurements for the quantification of edema in human brain tissue - Open-ended coaxial and coplanar probes for fast tissue scanning, IEEE Sensors '14, pp. 681-683, 2014.

[16] R. N. Simons, Coplanar Wavequide Circuits, Components and Systems, John Wiley \& Sons, 2001.

[17] Nicolson, A.M.; Ross, G. F., "Measurement of the Intrinsic Properties of Materials by Time-Domain Techniques," Instrumentation and Measurement, IEEE Transactions on , vol.19, no.4, pp.377,382, 1970.

[18] Baker-Jarvis, J.; Vanzura, E.J.; Kissick, W.A., "Improved technique for determining complex permittivity with the transmission/reflection method," Microwave Theory and Techniques, IEEE Transactions on , vol.38, no.8, pp.1096,1103, 1990

[19] Cartwright G, McManus BH, Leffler TP, Moser CR. Rapid determination of moisture/solids and fat in dairy products by microwave and nuclear magnetic resonance analysis. J AOAC Int 2005;88:107-20. 